\title{
Editorial for the special issue on "Intelligent Computing and System towards Smart Manufacturing"
}

\author{
Stefan Wolfgang Pickl ${ }^{1} \cdot$ Bo Tao $^{2} \cdot$ T. W. Liao ${ }^{3} \cdot \mathrm{Fei} \mathrm{TaO}^{4}$
}

Published online: 15 October 2018

c) Springer-Verlag GmbH Germany, part of Springer Nature 2018

With the integration and extensive applications of the new generation information technologies (such as cloud computing, internet of things, big data, mobile internet, artificial intelligence) in manufacturing industry, a number of countries have put forward their national advanced manufacturing development strategies, such as Industry 4.0 in Germany, Industrial Internet and manufacturing system based on cyber-physical systems (CPS) in the USA, as well as Made in China 2025 and Internet Plus Manufacturing in China. Although each of these strategies was proposed under different circumstances, one of the common aims is to achieve smart manufacturing. However, without the support of data and intelligent computing technologies, "smart" cannot be achieved.

The objective of the special issue on "Intelligent Computing and System towards Smart Manufacturing" is to present the latest advances and developments of methods, technologies, systems, and tools dedicated to the intelligent computing science and technology toward smart manufacturing. Under the support of related worldwide researchers, 45 papers have

\footnotetext{
Fei Tao

ftao@buaa.edu.cn

Stefan Wolfgang Pickl

stefan.pickl@unibw.de

Bo Tao

taobo@hust.edu.cn

T. W. Liao

ieliao@1su.edu
}

1 Department of Computer Science, COMTESSA, Universität der Bundeswehr München, Neubiberg, Germany

2 School of Mechanical Science and Engineering, Huazhong University of Science and Technology, Wuhan 430074, China

3 Department of Mechanical and Industrial Engineering, Louisiana State University, Baton Rouge, LA 70803, USA

4 School of Automation Science and Electrical Engineering, Beihang University, Beijing 10091, China been received. Based on the review comments from peer reviewers, 33 papers have been selected out for the special issue and authors have revised their paper according to the comments before the final acceptance. The 33 paper which cover broad topics are introduced briefly as follows.

In these papers, intelligent algorithms have been applied in analysis, evaluation and optimization in different fields to obtain optimal solutions and data-based intelligence.

The paper "Deep learning based smart radar vision system for object recognition" presented a novel and robust data-driven vision system for recognizing different types of objects in various external situations based on deep learning algorithms. The proposed system achieves better performances than other systems in experiments.

The paper "An effective soft computing technology based on belief-rule-base and particle swarm optimization for tipping paper permeability measurement" proposed a soft computing technology for the tipping paper permeability measurement in a tobacco factory. To improve the accuracy of the computing result, the paper studied the structure and parameters optimization of Belief Rule Base and a particle swarm optimization algorithm with improved velocity update way and repair methods.

A new deep neural network model based on GaussianBernoulli deep Boltzmann machine was presented for mechanical condition prognosis in the paper "Deep Boltzmann machine based condition prediction for smart manufacturing". The proposed method was applied in the condition prediction of a compressor.

The paper "Some Single-Valued Neutrosophic Bonferroni Power Aggregation Operators in Multiple Attribute Decision Making" developed single-valued neutrosophic Bonferroni power aggregation operators and single-valued neutrosophic geometric Bonferroni power aggregation operators to solve the multiple attribute decision making problem.

In the paper "Revenue sharing model in new Hong Kong's warehousing business paradigm", a new paradigm for Hong Kong's warehousing industry was proposed by 
introducing a third-party warehousing equipment supplier and then a stackelberg game was played to study stakeholders' optimal strategies.

In the paper "Manipulator multi-objective motion optimization control for high voltage power cable mobile operation robot", an $n$-order improved polynomial interpolation trajectory algorithm was proposed to achieve excellent trajectory performance.

In the paper "Dual-process modeling and control method for new product collaborative design based on Petri Net", a Petri Net was established to make the whole design process in a collaborative environment and to analyze design relations among the design majors from the perspective of fine granularity.

In the paper "An approach to predict discharge voltage of lithium-ion batteries under dynamic loading conditions", the problem of predicting the terminal voltage was transformed into the problem of predicting the state-of-charge of battery, and then an adaptive capacity method based on feature extraction and k-nearest neighbor algorithm was developed to support the prediction.

In the paper "Comparison of series products from customer online concerns for competitive intelligence", a framework of representative sentence sampling from online reviews of series products was outlined, and a greedy algorithm was designed to obtain the sampling results efficiently.

In the paper "Research on ECBOM modeling and energy consumption evaluation based on BOM multi-view transformation", the concept of energy consumption bill of materials (ECBOM) was firstly put forward, then an UML conceptual model of the ECBOM and a traversal algorithm were designed to efficiently search data for energy consumption evaluation.

In addition, some innovative intelligent optimization algorithms are proposed to solve the single-objective/multiobjective optimization problem, including a fast algorithm based on score ranking in the paper "Energy-efficiency-oriented scheduling in smart manufacturing", a mutant firefly algorithm in the paper "Solving the two-stage hybrid flow shop scheduling problem based on mutant firefly algorithm", an improved ant colony algorithm in the paper "Wireless sensor network routing method based on improved ant colony algorithm", and a polynomial-time algorithm $H$ in the paper "Two-agent scheduling on a single parallel-batching machine to minimize the weighted sum of the agents' makespans".

As the new generation information technologies can endow the manufacturing process with intelligence, some papers have integrated these technologies with manufacturing and explored the advanced manufacturing modes.

The paper "Fog manufacturing paradigm: architecture, control mechanism, classification, modeling and testing platform" proposed the fog manufacturing concept based on the development of CPS, digital twin, fog computing and edge computing, then compared the fog manufacturing with other manufacturing modes using the analytic hierarchy process method, and finally built a testing platform for verification.

In the paper "IoT-enabled dynamic lean control mechanism for typical production systems", a Lean-Oriented Optimum-State Control Theory (L-OSCT) was put forward and then applied in the dynamic process control based on the IoT-driven synchronization method.

The paper "A grey correlation based supply-demand matching of machine tools with multiple quality factors in cloud manufacturing environment" proposed modeling methods for manufacturing demands and cloud services, and constructed a mapping mechanism between them. Based on this, a supply-demand matching method was put forward using the grey correlation theory.

In the paper "a metadata based manufacturing resource ontology modeling in cloud manufacturing systems", a metadata-based modeling method was presented to keep descriptions of manufacturing resources consistent in the cloud environment, thus shielding the heterogeneity.

In the paper "A cloud-terminal-based cyber-physical system architecture for energy efficient machining process optimization", an innovative four-layer cloud-terminal-based cyber-physical system architecture including the machine level, control level, data level and decision support level was presented to optimize the machining process considering energy efficiency.

In the paper "Selection of outsourcing services for cement equipment manufacturing enterprises in cloud manufacturing", the authors built a novel heuristic approach for service discovery and combinatorial optimization of outsourcing resources. Experimental results showed that the proposed method is more efficient for solving large-scale problems in cloud manufacturing environment.

In the paper "A knowledge generation mechanism of machining process planning using cloud technology", a knowledge generation mechanism was proposed to produce machining solutions through taking advantages of Map/ Reduce framework for large-scale data handling.

In the paper "A framework for shopfloor material delivery based on real-time manufacturing big data", key technologies for a material delivery framework were investigated, including data sensing and acquisition, big data preprocessing and storage, as well as manufacturing big data mining.

In the paper "Research and development of off-line services for the 3D automatic printing machine based on cloud manufacturing", an off-line 3D automatic printing machine method based on cloud manufacturing service mode and a 3D printing cloud service platform were built to improve the competitiveness of 3D printing technologies.

Digital twin as an emerging technology to realize digitalization of the physical world and fusion between the physical 
and virtual worlds has attracted a lot of attentions in both academic and industry. Some papers have applied the digital twin concept in the manufacturing to obtain efficiency improvements and economic benefits through modeling and simulation, iterative interaction and optimization, data integration and fusion, etc.

In the paper "Digital twin for CNC machine tool: modeling and using strategy", a modeling and application framework for the digital twin of a CNC machine tool was proposed firstly, then implemented based on the design of descriptive model, mapping strategy and algorithm model.

In the paper "An application framework of digital twin and its case study", the authors proposed a three-layer application framework of the digital twin for product lifecycle management and constructed a digital twin system of a welding production line in the case study to improve product quality and production efficiency.

In the paper "Digital twin-driven manufacturing cyberphysical system for parallel controlling of smart workshop", cyber-physical connections via decentralized digital twin models were established to form a dynamic autonomous system of manufacturing resources for producing personalized products. Characteristics, architecture, configuration, operating mechanism and key enabling technologies of the proposed system were elaborated.

In the paper "Parameterized representation and solution method of the lightweight 3D model virtual assembly constraint", a modeling method for the lightweight 3D model was proposed to lay a foundation for the realization of digital twin in the field of smart manufacturing.

In the paper "Modular based flexible digital twin for factory design", the authors combined the digital twin with factory design to enable quick design evaluation and fault detection.

In the paper "Research on modelling and optimization of hot rolling scheduling", digital twin was used to optimize the scheduling results iteratively to drive the hot rolling production line.

In the paper "A simulation-based approach for plant layout design and production planning", a framework and an implementation procedure were developed for the simulation-based plant layout design and production planning. A case study showed that the proposed framework could be easily followed by companies.

The paper "Modeling and simulation of sheet-metal part deformation in virtual assembly" presented a new method based on the triangle facet model and the Laplace operator to calculate the deformation of a sheet-metal part during an assembly process simulation. Compared with the Finite Element Method, the proposed method has a higher solving speed and a simpler preprocessing process.

The paper "Intelligent design based on holographic model using parametric design method" proposed a product-level parametric management and rapid design method based on the holographic modeling and developed a parametric design system for the compensator product in the case study.

The paper "A rapid virtual assembly approach for 3D models of production line equipment based on the smart recognition of assembly features" put forward a 3D-2D-3D assembly feature recognition method to transform an original 3D model into a lightweight 3D model for the rapid virtual assembly.

This special issue has been made possible by the strong support of Prof. Vincenzo Loia, the Editor-in-Chief of the Journal of Ambient Intelligence and Humanized Computing. We would like to express our gratitude to the authors for their contributions, the referees for ensuring the quality of the accepted papers, and the staff of the Editorial Office and Production Department for their help in finalizing this special issue.

Publisher's Note Springer Nature remains neutral with regard to jurisdictional claims in published maps and institutional affiliations. 